\title{
Defective Major Histocompatibility Complex Class I Expression on Lymphoid Cells in Autoimmunity
}

\author{
Yineng Fu, David M. Nathan, Fangqin Li, Xiangping Li, and Denise L. Faustman \\ Immunobiology Laboratories of the Diabetes Unit and Medical Services, Massachusetts General Hospital \\ and the Department of Medicine, Harvard Medical School, Boston, Massachusetts 02114
}

\begin{abstract}
Lymphocytes from patients with insulin-dependent diabetes mellitus (IDDM), a chronic autoimmune disease, have recently been shown to have decreased surface expression of MHC class I antigens. Since IDDM and other autoimmune diseases share a strong genetic association with MHC class II genes, which may in turn be linked to genes that affect MHC class I expression, we studied other autoimmune diseases to determine whether MHC class I expression is abnormal. Fresh PBLs were isolated from patients with IDDM, Hashimoto's thyroiditis, Graves' disease, systemic lupus erythematosis, rheumatoid arthritis, and Sjogren's syndrome. Nondiabetic and non-insulin-dependent diabetes mellitus patients served as controls. MHC class I expression was measured with a conformationally dependent monoclonal antibody, W6/32. Freshly prepared PBLs from the autoimmune diseases studied and the corresponding fresh EBV-transformed B cell lines had decreased MHC class I expression compared with PBLs from normal volunteers and non-insulin-dependent (nonautoimmune) diabetic patients. Only 3 of more than 180 donors without IDDM or other clinically recognized autoimmune disease had persistently decreased MHC class I expression; one patient was treated with immunosuppressive drugs, and subsequent screening of the other two patients revealed high titers of autoantibodies, revealing clinically occult autoimmunity. Patients with nonautoimmune inflammation (osteomyelitis or tuberculosis ) had normal MHC class I expression. Autoimmune diseases are characterized by decreased expression of $\mathrm{MHC}$ class I on lymphocytes. MHC class I expression may be necessary for self-tolerance, and abnormalities in such expression may lead to autoimmunity. (J. Clin. Invest. 1993. 91:23012307.) Key words: major histocompatibility complex-class I • insulin-dependent diabetes mellitus • Graves' disease • Hashimoto's thyroiditis • systemic lupus erythematosis • rheumatoid arthritis
\end{abstract}

Address reprint requests to Dr. Denise Faustman, Immunobiology Labs of the Diabetes Unit, MGH East, Bldg. 149, 13th Street, Charlestown, MA 02129.

Received for publication 3 June 1992 and in revised form 21 January 1993

J. Clin. Invest.

(C) The American Society for Clinical Investigation, Inc.

$0021-9738 / 93 / 05 / 2301 / 07 \quad \$ 2.00$

Volume 91, May 1993, 2301-2307

\section{Introduction}

Insulin-dependent diabetes mellitus (IDDM) ${ }^{1}$ is characterized by autoimmune destruction of the insulin-producing beta cells of the pancreas (1). Similar to other autoimmune diseases, IDDM has a genetic predisposition. In the absence of an identified specific gene abnormality, the strongest genetic associations have been between IDDM and genes encoding the MHC class II polypeptides (2-4). Similar linkage has been shown for other autoimmune diseases, including Graves' disease, Hashimoto's thyroiditis, systemic lupus erythematosis, and autoimmune adrenal insufficiency (5-7). MHC class II molecules function to present foreign proteins to $\mathrm{T}$ lymphocytes. The strong association between autoimmune disease and MHC class II genes has suggested that abnormalities in MHC class II gene products play a central pathogenetic role in autoimmune diseases.

Our recent work in IDDM has shown that in human (and murine) autoimmune diabetes, antigen presentation and expression of the MHC class I pathway is defective (8). Moreover, complete or partial transgenic disruption of MHC class I expression alone is sufficient to induce a syndrome of mild autoimmune diabetes in mice that are otherwise not susceptible to diabetes. Finally, preliminary evidence in a mouse model of spontaneous autoimmune diabetes (the nonobese diabetic [NOD] mouse) links the defective expression of MHC class I molecules to genes located within the MHC class II region (8); these genes within the MHC class II region code for proteins that control endogenous peptide delivery into the endoplasmic reticulum and allow proper MHC class I assembly and subsequent MHC class I cell surface expression ( 8-16). Interruption of these MHC class II-linked genes uniformly results in lowered MHC class I expression on lymphocytes. The central role of MHC class I expression and disease course is well illustrated in the NOD diabetic mouse as well as in identical twins discordant for IDDM. Independent of genotype, only the subset of NOD mice with decreased MHC class I expression develops diabetes. In identical twins discordant for IDDM, only identical twins with IDDM have reduced MHC class I expression on peripheral blood lymphocytes; the nondiabetic twins have normal or near normal MHC class I expression. From this work, it appears that proper MHC class I presentation may be a pathway for self-tolerance and if altered, regardless of genotype, can lead to autoimmune diabetes.

1. Abbreviations used in this paper: IDDM, insulin-dependent diabetes mellitus; MAD, mean antigen density; NIDDM, non-insulin-dependent diabetes; NOD, nonobese diabetic. 
Because of the strong association of autoimmune diabetes (IDDM) with the MHC class I defect, we investigated other autoimmune diseases for defective MHC class I expression on fresh peripheral blood as well as on freshly produced EBVtransformed B cell lines.

\section{Methods}

Patients. Patients with IDDM $(n=54)$, Graves' disease $(n=36)$, Hashimoto's thyroiditis $(n=16), \operatorname{SLE}(n=14)$, and RA $(n=16)$ were identified from the out-patient clinics of the Massachusetts General Hospital. To be included, patients had to have histories and physical findings consistent with their diagnoses. Additionally, for Graves' disease, Hashimoto's thyroiditis, SLE, and RA, serological evidence of the specific autoimmune disease (long-acting thyroid stimulating, antithyroid, and anti-DNA antibodies, and rheumatoid factor, respectively) was required. Patients with IDDM had diabetes onset before age 30, were ketosis prone, and were treated continuously with insulin. Normal volunteers and patients with non-insulin-dependent diabetes mellitus (NIDDM) who had no personal history and no family history of any of the diseases listed above were included as control subjects. NIDDM patients $(n=42)$ developed their diabetes after age 45 and had no history of urine ketones. One NIDDM patient was on high dose steroids for kidney transplant. Five lupus patients, one Sjögren's patient, and two rheumatoid arthritis patients were treated with low dose steroids (generally less than $10 \mathrm{mg}$ Prednisone daily) for their underlying disease process. Seven patients with nonautoimmune inflammation ( six with osteomyelitis and one with tuberculosis) were studied as an additional control group. Five of the six patients with osteomyelitis also had NIDDM.

Study design. A preliminary study on MHC class I expression in fresh peripheral blood lymphocytes from controls ranging in age from 12 to $45 \mathrm{yr}$ revealed no age, sex, or HLA haplotype-related changes in this marker. MHC class I expression was slightly age related between ages 2 and 12, therefore IDDM patients of this age group were analyzed with age-matched controls. Patients with the other autoimmune diseases were all older than $12 \mathrm{yr}$. A comparison of the results of repeated assays of MHC class I expression on controls performed on the same day consistently revealed $<1 \%$ absolute differences in percentage $(95 \%$ confidence interval) and $<2 \%$ absolute difference $(95 \%$ confidence interval) in mean antigen density between repeated analyses of the same sample or between different normal individuals who were analyzed for MHC class I antigens using either MHC class I antibodies (W6/32, BBM1) or antibody directed to $\beta_{2}$-microglobulin, the cell surface protein which associates in a 1:1 fashion with MHC class I. An example of the histograms of MHC class I expression for eight normal donors, measured on the same day, is shown in Fig. 1. In contrast, greater variation was seen in the same normal sample analyzed on different days due to the well-described analysis day effect of flow cytometry. An analysis of covariance with terms for age, sex, group ( same control sample compared with repetitive self-sample versus another control sample), HLA type, and analysis day, revealed only a significant effect of analysis day $(P=0.0004)$. The results of this preliminary study dictated that isolated peripheral blood lymphocytes from patients and control subjects be obtained and analyzed on the same day. Patient and control EBV lines were also analyzed on the same day for direct comparison. This study design was also chosen to prevent the changes in lymphocyte surface markers that occur with incubation, freezing, and fixation $(17,18)$. A paired $t$ test was used to test whether lymphocyte surface markers (MHC class I) were different between patients in each group and their paired control subjects. Data are reported as mean \pm standard deviation unless noted otherwise.

Flow cytometer analysis. PBLs were prepared, as previously described, from $40 \mathrm{cc}$ of blood freshly collected in heparinized tubes (17). In addition to the analysis of PBLs, new permanent $B$ cell lines were established by EBV transformation of fresh B cells from both patients

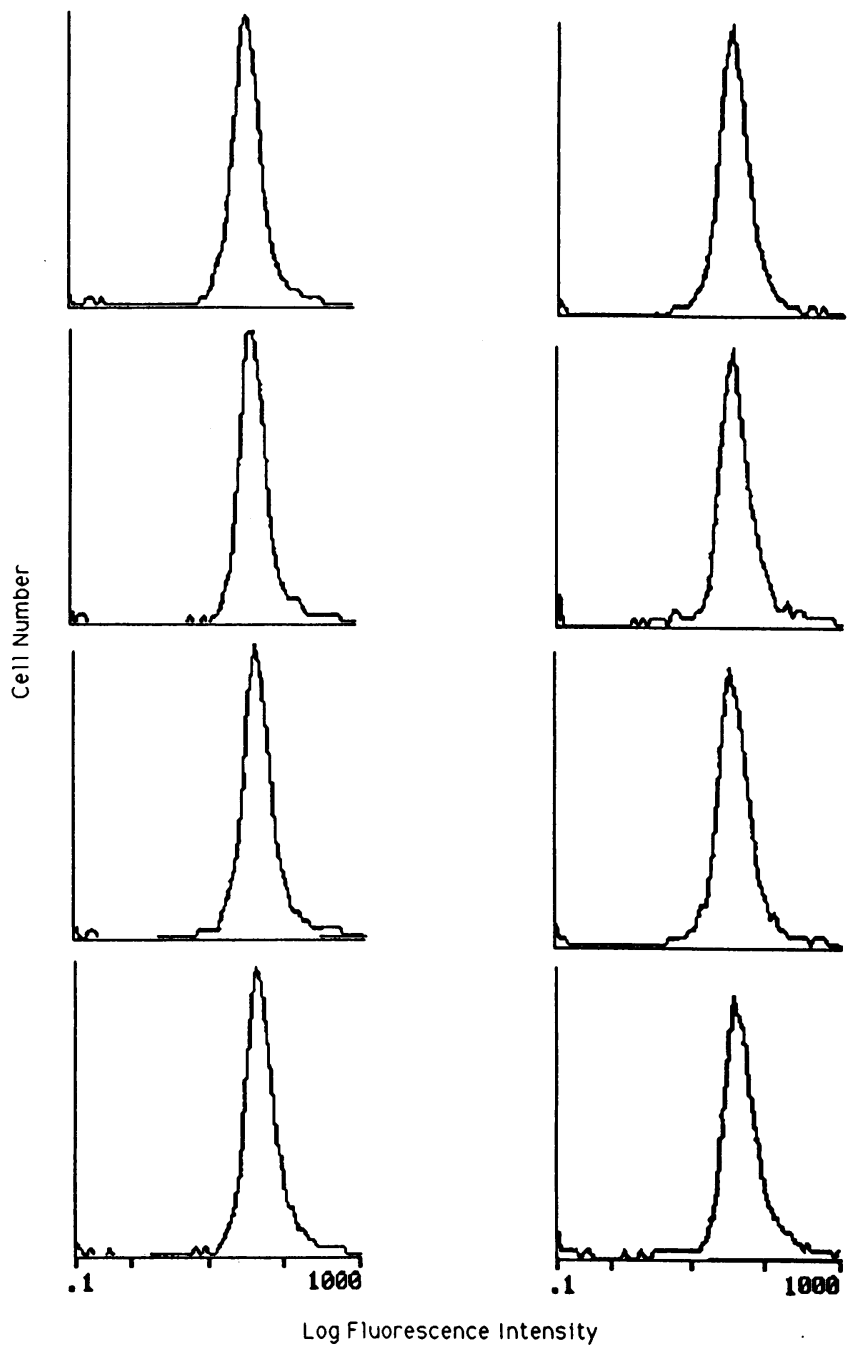

Figure 1. MHC class I expression on PBLs from eight normal donors analyzed on the same day. PBLs were analyzed with $\mathrm{W} 6 / 32$, a monoclonal antibody to human class $I$.

and controls. Approximately 2-3 mo after EBV transformation, the newly transformed patient cell lines were randomly paired with newly transformed EBV cell lines from control individuals and analyzed by immunofluorescence. Most new patient EBV cell lines were analyzed at least twice with different simultaneously paired new control EBV cell lines. Patient and control cell lines appeared stable during the time frame of this study; the data presented represent data from only one of the analysis days. Fluorescence activated cell sortic analysis was used to quantitate cell surface differences in class I density. This technique was chosen because of the ability of this method to quantitate accurately epitope density over four logs of immunofluorescence.

For the analysis of MHC class I determinants on fresh cells or EBV lines, mouse monoclonal antibody W6/32 (American Type Culture Collection, Rockville, MD), which recognizes all alleles of human MHC class I, was used at a fresh stock concentration of $0.8 \mu \mathrm{g} / \mathrm{ml}$ with the addition of 100 microliters for $1 \times 10^{6} \mathrm{EBV}$ cells or fresh stock at $1.6 \mu \mathrm{g} / \mathrm{ml}$ with the addition of $100 \mu \mathrm{l}$ for $1 \times 10^{6} \mathrm{PBLs}$. This concentration of antibody represents an excess over the $50 \%$ saturation point for each cell population but eliminates irrelevant $\mathrm{Fc}$ receptor binding when used at a higher concentration or low-affinity binding to the class I dimer, i.e., class I and $\beta_{2}$-microglobulin without peptides. The purified W6/32 antibody was used with goat anti-mouse IgG2a FITC (Tago, Burlingame, CA) for indirect immunofluorescence at a concen- 
Table I. Expression of MHC Class I Determinants on Freshly Isolated Lymphocytes from a Variety of Autoimmune Diseases

\begin{tabular}{lccc}
\hline & $\begin{array}{c}\text { No. of } \\
\text { paired } \\
\text { samples }\end{array}$ & \multicolumn{2}{c}{$\begin{array}{c}\text { Expression of MHC } \\
\text { class I antigens* }\end{array}$} \\
\hline & & $\%$ & $M A D$ \\
IDDM control & 54 & $82.4 \pm 13$ & $6.0 \pm 4.5$ \\
& & $90.9 \pm 12$ & $9.7 \pm 6.7$ \\
NIDDM control & 42 & $P<0.001$ & $P<0.001$ \\
& & $97.4 \pm 2.7$ & $15.5 \pm 7.6$ \\
& & $96.6 \pm 3.4$ & $16.3 \pm 8.4$ \\
Hashimoto's control & 16 & $77.7 \pm 21$ & $P=0.37$ \\
& & $94.5 \pm 3.1$ & $10.9 \pm 5.6$ \\
Graves' control & \multirow{2}{*}{36} & $P=0.003$ & $P=0.001$ \\
& & $79.5 \pm 22$ & $7.4 \pm 4.5$ \\
Lupus control & & $91.4 \pm 14$ & $12.3 \pm 7.2$ \\
& \multirow{2}{*}{14} & $8<0.001$ & $P<0.001$ \\
& & $95.4 \pm 2.5$ & $14.4 \pm 4.5$ \\
Rheumatoid arthritic & & $P=0.01$ & $P=0.009$ \\
control & & & \\
& \multirow{2}{*}{17} & $80.4 \pm 18$ & $10.0 \pm 11.5$ \\
& & $93.8 \pm 7.7$ & $13.1 \pm 11.6$ \\
& & $P=0.01$ & $P=0.02$ \\
& & &
\end{tabular}

* Values are mean \pm standard deviation for a paired $t$ test between a patient and simultaneously paired control samples. Patients less than $12 \mathrm{yr}$ old also had an age-matched paired sample due to the slight differences in MHC class I expression in younger children.

tration greater than 100 -fold excess of the $50 \%$ saturation point. The W6/32 antibody was selected because it is conformationally dependent and thus demonstrates most dramatically the lowered MHC class I on autoimmune cells (19). Subset analysis of fresh peripheral blood lymphocytes was performed by two-color analysis using directly FITCconjugated W6/32 antibody with directly phycoerythrin (PE)-conjugated B1 antibody (B cell marker), Mo2 antibody (macrophage and monocyte marker), or CD2 antibody ( $\mathrm{T}$ cell marker) (Coulter Electronics Inc., Hialeah, FL). For two-color analysis, FITC emission was separated from PE emission using appropriate filters, differentially conjugated beads, as well as a control sample labeled with CD4 and CD8 for the determination of the appropriate compensation. All phenotyping samples were analyzed on a flow cytometer (Elite; Epic Inc., New York); more than 5,000 cells per sample were analyzed. The sample flow rate was maintained at more than 350 cells/second. The operator was masked to sample identity. For PBL, a gate on the FACS ${ }^{\circledast}$ machine (Becton Dickinson \& Co., Mountain View, CA) was set to exclude small nonlymphocyte debris using a T200 antibody (KC57; Coulter Immunology) specific for all lymphocyte subpopulations. In addition, viable cells were identified by forward light scatter intensity and exclusion of propidium iodine which labels dead cells. For EBV analysis, a gate was set to exclude debris and included EBV cells of the large size on log fluorescence. EBV cells before analysis were in log phase of growth, never frozen and determined to have viability of $>95 \%$. The percentage of positive cells was determined using a three $\log$ decade histogram with a $95 \%$ region for isotype control. Background immunofluorescence, determined by an isotype-matched, FITC-conjugated, or PE-conjugated antibody was virtually identical in all cases and always less than $5 \%$.

Due to the difference in mean antigen density (MAD) of MHC class I on monocytes versus lymphocytes, two gates were set to analyze unseparated PBLs with single-color immunofluorescence using the W6/32 antibody. For data calculations in Tables I and II, a gate was set on the small-sized but abundant nonmonocyte population. As expected, identical statistical trends were observed for the data in Tables I and II with a gate set only on the very large-sized but less abundant monocyte subpopulation (data not shown). For the two-color immunofluorescence presented in Table III, an open gate excluding only nonlymphocyte debris was utilized. This open gate was used to determine the density of MHC class I on specific lymphocyte subpopulations, i.e., $\mathrm{T}$ cells, $\mathrm{B}$ cells, and monocytes.

\section{Results}

As previously reported, MHC class I antigen expression on fresh peripheral blood lymphocytes from patients with IDDM was consistently depressed (Table I, Fig. 2). Lymphocytes from NIDDM patients, on the other hand, had a similar mean percentage of MHC class I determinants compared with nondiabetic controls ( $97.4 \pm 2.7$ vs. $96.6 \pm 3.4, P=0.20$ ) (Table I) as expected for a disease without autoimmune etiology. In addition, fresh lymphocytes from NIDDM patients had a similar density of MHC class I antigens compared with fresh control cells ( $15.5 \pm 7.6$ vs. $16.3 \pm 8.4, P=0.37$ ). Interestingly, 3 of the 42 NIDDM patients analyzed appeared to have significant decreases in MHC class I percentages or mean antigen densities compared with simultaneously analyzed control samples. As outlined in Methods, normal control samples from different donors analyzed on the same day demonstrated less than $\pm 1 \%$ variability between individuals in percentage and/or less than $\pm 2 \%$ variability in mean antigen density of MHC class I antigens. If the absolute difference in percentage of MAD be-

Table II. Modification of NIDDM Lymphocyte MHC Class I Expression by Underlying Autoimmunity or Steroids

\begin{tabular}{|c|c|c|c|c|c|c|}
\hline & NIDDM Patient $1^{*}$ & Control & NIDDM Patient $2^{\ddagger}$ & Control & NIDDM Patient $3^{5}$ & Control \\
\hline \multicolumn{7}{|c|}{ Fresh PBL } \\
\hline$\%$ & $93 \%$ & $99 \%$ & $78 \%$ & $93 \%$ & $65 \%$ & $94 \%$ \\
\hline MAD $^{\|}$ & 7.08 & 17.7 & 7.2 & 13.5 & 9.3 & 18.2 \\
\hline \multicolumn{7}{|c|}{ EBV cell line } \\
\hline$\%$ & $97 \%$ & $96 \%$ & $42 \%$ & $82 \%$ & $97 \%$ & $99 \%$ \\
\hline MAD & 5.6 & 4.7 & 1.39 & 4.50 & 13.7 & 19.8 \\
\hline
\end{tabular}

* NIDDM patient on chronic high dose steroids secondary to a kidney transplant. ${ }^{\ddagger}$ NIDDM patient subsequently found to have anti-smooth muscle antibodies. ${ }^{\S}$ NIDDM patient subsequently found to have anti-thyroid colloid antibodies, antimicrosomal antibodies and antinuclear antibodies. "Mean antigen density. 
Table III. MHC Class I Expression on Subsets of Peripheral Blood Lymphocytes from Various Autoimmune Diseases

\begin{tabular}{|c|c|c|c|c|c|c|}
\hline & \multicolumn{2}{|c|}{ Macrophage/monocyte* } & \multicolumn{2}{|c|}{ B cells* } & \multicolumn{2}{|c|}{ T cells* } \\
\hline & $\%$ & $M A D$ & $\%$ & $M A D$ & $\%$ & $M A D$ \\
\hline \multicolumn{7}{|l|}{ A. Individual patients } \\
\hline \multirow[t]{2}{*}{ IDDM patient ${ }^{\S}$ control } & 97 & 7.8 & 56 & 4.1 & 91 & 5.6 \\
\hline & 99 & 26 & 94 & 14 & 97 & 7.1 \\
\hline \multirow[t]{2}{*}{ Graves' patient control" } & 95 & 26 & 92 & 20 & 99 & 19 \\
\hline & 100 & 32 & 100 & 27 & 100 & 21 \\
\hline \multirow[t]{2}{*}{ Hypothyroid patient control } & 96 & 18 & 96 & 28 & 99 & 13 \\
\hline & 99 & 41 & 99 & 35 & 99 & 20 \\
\hline \multirow[t]{2}{*}{ Lupus patient control } & 90 & 33 & 84 & 28 & 90 & 14 \\
\hline & 96 & 39 & 86 & 31 & 92 & 25 \\
\hline \multirow[t]{2}{*}{ Sjogren's patient control } & 97 & 19 & 96 & 24 & 98 & 18 \\
\hline & 99 & 56 & 99 & 41 & 100 & 30 \\
\hline \multicolumn{7}{|c|}{ B. Combined autoimmune patients ${ }^{\ddagger}$} \\
\hline & \multicolumn{2}{|c|}{$M A D$} & \multicolumn{2}{|c|}{$M A D$} & \multicolumn{2}{|c|}{$M A D$} \\
\hline \multicolumn{7}{|l|}{ Autoimmune patients $n=53$} \\
\hline \multirow[t]{2}{*}{ Controls } & \multicolumn{2}{|c|}{$32 \pm 2.7$} & \multicolumn{2}{|c|}{$24 \pm 1.9$} & \multirow{2}{*}{\multicolumn{2}{|c|}{$\begin{array}{c}17 \pm 1.2 \\
P=0.000\end{array}$}} \\
\hline & \multicolumn{2}{|c|}{$P=0.000$} & \multicolumn{2}{|c|}{$P=0.000$} & & \\
\hline
\end{tabular}

* As described in Methods, two-color immunofluorescence was performed, using directly conjugated W6/32 (MHC class I monoclonal marker) with Mo2 (macrophage and monocyte monoclonal marker), B1 (B cell monoclonal marker), or CD2 (T cell monoclonal marker) to quantitate the relative amounts of MHC class I epitopes on different lymphocyte subsets. ${ }^{\ddagger}$ These data represent pooled values from 10 lupus patients, 15 Graves' disease patients, 8 rheumatoid arthritis patients, 10 Hashimoto's patients, and 10 type I diabetics compared with their paired controls. ${ }^{\S}$ As discussed in Methods, the mean antigen density of MHC class I on lymphocytes does not vary between adults age $12-45$ yr old. In contrast, MHC class I on lymphocytes from children, ages 2-12 yr old, is age dependent. Many of our IDDM patients were children and were age matched, therefore accounting for the slightly lower mean values for the density of MHC class I on the patients and matched controls in this autoimmune category of patients. "Because of the log fluorescence scale for mean antigen density and the fact that the total number of class I molecules is less on T cells compared with B cells or macrophages, the magnitude of class I change was usually least dramative in the T cell subset. This is well demonstrated in the above table for the Graves' disease patient where the $T$ cell fluorescence is decreased in the patient but the actual values are close to the control value.

tween the patient and control exceeded $1 \%$ or $2 \%$ respectively, the patient was considered to have abnormal MHC class I expression. Three NIDDM patients demonstrated an unexpected decrease $(6 \%, 15 \%$, and $29 \%)$ in percentage and decreased in mean antigen density $(11 \%, 6.3 \%$, and $9 \%)$ of MHC class I on lymphocytes compared with their nondiabetic controls. Further studies were performed in these NIDDM patients. One of the three NIDDM patients was treated with high dose glucocorticoids as well as immunosuppressive drugs (Cyclosporin A; Sandoz, Basel, Switzerland; and Aziothioprine; Burroughs
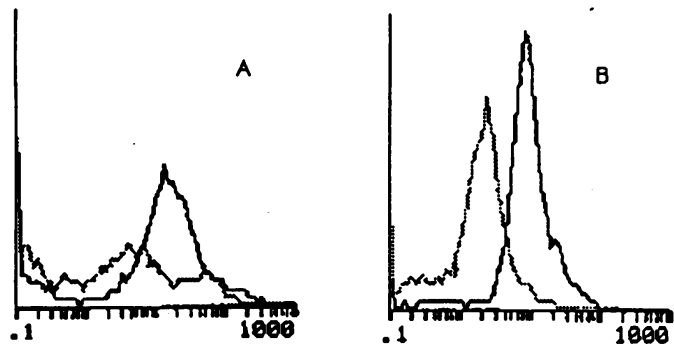

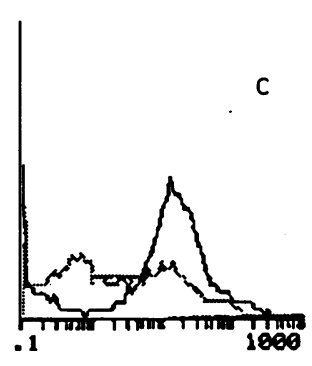

$\frac{\text { 苂 }}{\text { o }}$

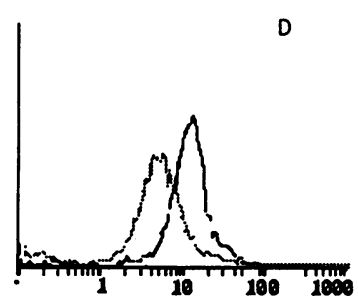

Fluorescence Intensity

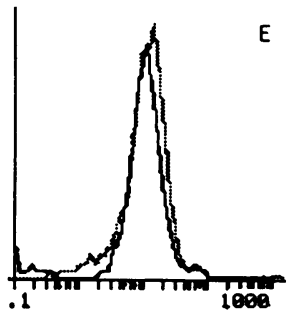


Wellcome, Research Triangle, NC) for a kidney transplant ( Table II, patient 1). Steroids and immunosuppressive drugs have known lymphocyte-modifying effects and therefore might modify MHC class I expression on fresh peripheral blood lymphocytes (20). Newly produced EBV transformed B cells from this patient demonstrated the return of normal MHC class I determinants in culture, demonstrating that the in vivo depression in MHC class I was most likely secondary to the in vivo drug effects (Table II). The other two NIDDM patients with low MHC class I expression on fresh lymphocytes were not treated with any medication that could adversely affect MHC class I determinants and had no clinical or family history of autoimmune diseases. A laboratory screen for clinically silent autoimmunity revealed high titers of anti-smooth-muscle antibodies in one patient and anti-thyroid-colloid antibodies, antimicrosomal antibodies, and antinuclear antibodies in the other patient, suggesting underlying autoimmunity was present in these two NIDDM patients identified by low MHC class I antigen expression on fresh peripheral blood lymphocytes. Newly established EBV cell lines from both NIDDM patients maintained decreased MHC class I expression with repeated phenotyping, as predicted for a genetically controlled autoimmune abnormality (Table II). The seven patients with osteomyelitis or tuberculosis had normal MHC class I expression with $99.3 \pm 0.59 \%$ expression and $13.5 \pm 3.3 \mathrm{MAD}$ and thus were virtually identical to the control samples performed on the same day as expected for a nonautoimmune but inflammatory disease.

We studied four groups of patients with other autoimmune diseases. The lymphocytes from 29 of 36 Graves' disease patients had a decreased percentage of MHC class I positive lymphocytes with a mean percentage of $79.5 \pm 1.22$ compared with $91.4 \pm 14$ percent positive fresh lymphocytes for the controls $(P$ $<0.001$ ) (Table I). Decreased expression was similarly reflected in the MAD of MHC class I epitopes on Graves' disease lymphocytes compared with control lymphoid cells $(7.4 \pm 45$ vs. $12.3 \pm 7.2, P<0.001$ ) (Table I).

The fresh lymphocytes from 12 of 14 lupus patients also had a decreased percentage of MHC class I positive lymphocytes compared with control lymphocytes $(81.2 \pm 20$ vs. $95.4 \pm 2.5 ; P=0.01$ ) (Table I); fresh lymphocytes from lupus patients also had a depression in the MHC class I density of MHC class I epitopes ( $7.4 \pm 4.5$ vs. $14.4 \pm 11.4)$ in 12 of 14 individuals compared with simultaneously analyzed controls $(P$ $=0.009$ ).

15 of 16 patients with Hashimoto's thyroiditis had fresh lymphocytes with decreased MHC class I determinants compared with the lymphocytes from matched controls $(77.7 \pm 21$ vs. $94.5 \pm 3.1, P=0.003)$; the mean density of MHC class I antigens was similarly depressed in these hypothyroid individuals ( $6.9 \pm 5.6$ vs. $10.0 \pm 7.7, P=0.001)$ ( Table I).

The fresh lymphocytes from 16 of 17 rheumatoid arthritis patients demonstrated a statistically significant depression in MHC class I expression for both the percentage of positive lymphocytes $(P=0.01)$ as well as mean antigen density $(P$ $=0.02)($ Table I $)$.

Peripheral blood lymphocytes represent a diverse variety of cell types, i.e., macrophages/monocytes, B cells, and T cells. These cells can be nonspecifically identified by flow cytometry analyses by their distinct sizes ( $T$ and $B$ cells vs. monocytes), as well as individually studied by two-color flow analysis with cell-specific antibodies in combination with a MHC class I an- tibody. Both of these studies were performed on fresh blood samples in order to determine if the defective MHC class I expression in fresh peripheral blood was confined to a given subpopulation of cells or expressed equally by all lymphoid cells. In each sample from patients with the autoimmune diseases, differential gating by cell size revealed similar, statistically significant, MHC class I depression in all cell populations compared with similarly gated fresh control cells. Follow-up two-color immunofluorescence, which finds specific lymphocyte cellular subsets, identified low MHC class I expression proportionally on T cells, B cells as well as macrophages/monocytes from type I diabetics, Graves', Hashimoto's/ hypothyroidism, lupus, and Sjögren's syndrome. Table III shows the results of this two-color analysis for five different diseases as well as for all autoimmune patients combined and demonstrates the nonexclusive nature of the MHC class I defect in different lymphocyte subpopulation from fresh blood samples of patients with diverse autoimmune diseases. Examples of individual histograms of the cellular subsets are shown in Fig. 3. The autoimmune histograms for each cellular subset, i.e., $\mathrm{T}$ cells $(A, B)$, B cells $(C, D)$ and macrophages $(E, F)$ clearly demonstrate a shift to the left, even when the $y$-axis fluorescence is represented on a log scale. Because the total number of class I molecules is less on $\mathrm{T}$ cells than $\mathrm{B}$ cells or macrophages, the magnitude of the log fluorescence change in class I density (fluores-
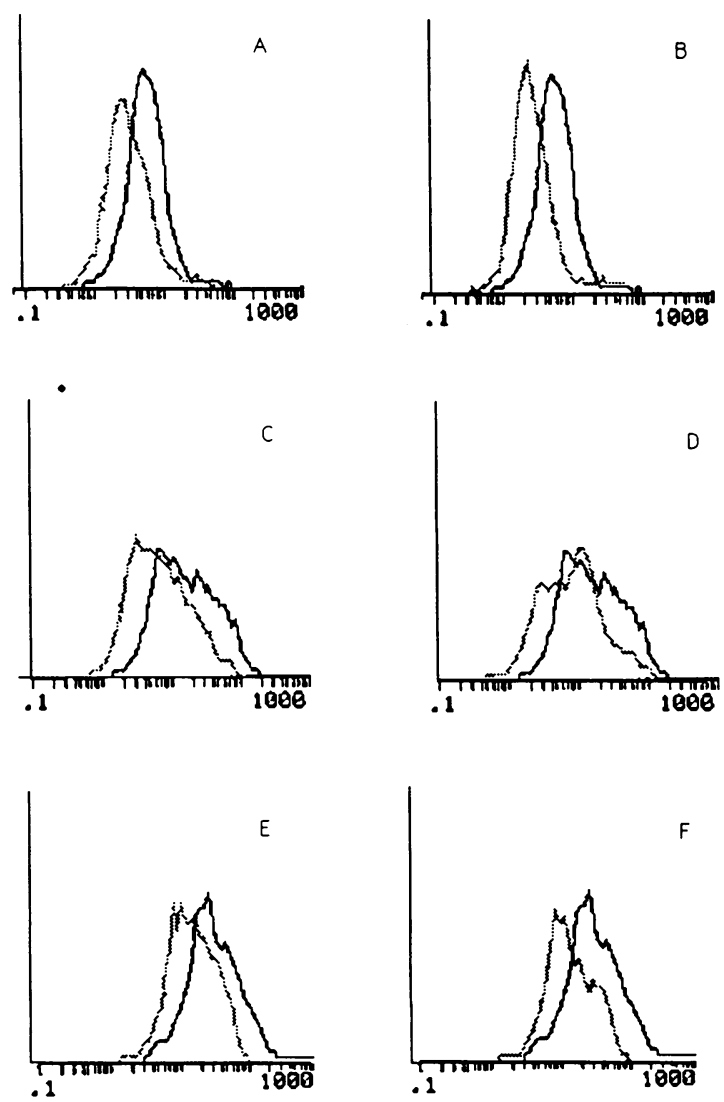

Figure 3. MHC class I expression on T cells, B cells, and macrophages from two type I diabetic patients (dashed lines; $A, C, E$ represent Patient 1 and $B, D, F$ represent Patient 2) compared with matched control (solid line). All samples were analyzed on the same day. MHC class I expression on T cells $(A, B)$, B cells $(C, D)$, and macrophages $(E, F)$ from the two different type I diabetic patients are shifted to the left compared with the control. 
Table IV. Expression of MHC Class I Determinants in EBV Cell Lines from a Variety of Autoimmune Diseases

\begin{tabular}{lccc}
\hline & $\begin{array}{c}\text { No. of } \\
\text { paired samples }\end{array}$ & \multicolumn{2}{c}{$\begin{array}{c}\text { Expression of MHC } \\
\text { class I antigens* }\end{array}$} \\
\hline & & $\%$ & $M A D$ \\
IDDM control & 35 & $76.7 \pm 16.0$ & $4.3 \pm 3.2$ \\
& & $92.6 \pm 6.7$ & $6.7 \pm 5.0$ \\
NIDDM control & 11 & $P<0.001$ & $P<0.001$ \\
& & $95.9 \pm 2.2$ & $7.1 \pm 3.2$ \\
Graves'/Hashimoto's & & $P=0.57$ & $P=0.80$ \\
control & & & \\
& & $84.6 \pm 18.9$ & $4.7 \pm 2.9$ \\
& & $96.3 \pm 5.3$ & $7.7 \pm 5.3$ \\
Lupus control & & $P=0.03$ & $P=0.02$ \\
& 7 & $59.1 \pm 31$ & $2.7 \pm 1.4$ \\
& & $88.0 \pm 8.8$ & $6.3 \pm 3.2$ \\
Rheumatoid arthritis & & $P=0.02$ & $P=0.004$ \\
control & & & \\
& 15 & $75.9 \pm 11.7$ & $3.8 \pm 1.5$ \\
& & $90.5 \pm 7.0$ & $7.8 \pm 2.8$ \\
& & $P<0.001$ & $P<0.001$ \\
& & &
\end{tabular}

* Values are mean \pm standard deviation for a paired $t$ test between a patient and simultaneously paired control samples. Patients less than $12 \mathrm{yr}$ old also had an age-matched paired sample due to the slight differences in MHC class I expression in younger children.

cence) was least impressive on $\mathrm{T}$ cells for all autoimmune categories of patients.

Nonconformationally dependent polyclonal anti- $\beta_{2}$-microglobulin serum was used on 10 IDDM fresh lymphocytes compared with 10 matched controls. Similar to the transporter mutant cell lines such as RMA-S, the magnitude of the lowered class I fluorescence was significantly less dramatic. The mean fluorescence for $\beta_{2}$-microglobulin was $37.5 \pm 6.7$ for IDDM cells; the mean fluorescence for $\beta_{2}$-microglobulin was $40.9 \pm 7.9$ for matched control $(P=0.16)$. Presumably because a conformationally dependent antibody such as class I specific W6/32 requires both the presence of class $I$ as well as peptide in the groove of class I for detection, the diabetic cells have both empty unstable class I on the cell surface as well as less total class I on the cell surface, thus explaining why conformationally dependent antibodies dramatize the class I defect better in autoimmune cells as well as on mutant cell lines.

As mentioned previously, many in vivo factors other than genetics could be influencing MHC class I expression. The possible influence of steroids and / or immunosuppression in modifying lymphocyte functions should be considered (20). To control for this variable, newly transformed EBV B cells were produced from patients for each of the autoimmune diseases. The analysis of these cell lines eliminated the possible influence of concurrent medications or yet unknown serum factors.

The percentage $(76.7 \pm 16$ vs. $92.6 \pm 6.7, P<.001)$ and mean fluorescence intensity $(4.3 \pm 3.2$ vs. $6.7 \pm 5.0, P<.001)$ of $\mathrm{MHC}$ class I determinants is depressed in type I diabetic EBV B-cell lines compared with control cell lines (Table IV). As expected, EBV cell lines from nonautoimmune NIDDM patients did not show depressed numbers of MHC class I cells (95.9 \pm 2.2 vs. $96.5 \pm 2.9, P=0.57$ ) or a difference in cell surface density of MHC class I determinants $(7.1 \pm 3.2$ vs. $6.9 \pm 2.9, P=0.80)$. All 3 Graves' EBV cell lines, all 7 lupus EBV cell lines, all 15 rheumatoid arthritis EBV cell lines, and the cell line established from a patient with Sjogren's all showed a decrease in the percentage ( $P=0.03, P=0.02, P=0.001$, respectively) as well as mean channel fluorescence $(P=0.02, P=0.004, P<0.001$, respectively) of MHC class I lymphocyte epitopes compared with simultaneously analyzed EBV cell lines from controls ( Table IV). Fig. 4 shows the characteristic histograms obtained from EBV cell lines analyzed with the monoclonal antibody directed to MHC class I for a given autoimmune disease compared with simultaneously matched control EBV cell line. For each autoimmune disease, the leftward shift in MHC class I fluorescence intensity reflects a decreased density of MHC class I epitopes in each transformed B cell line and thus decreased percentage of positive cells compared with controls. At no time during our analysis of over 80 new EBV cell lines from autoimmune patients did we observe maintenance of a normal MAD of MHC class I determinants with a decreased percentage of MHC class I positive cells; therefore, the existence of a truly negative MHC class I B lymphocyte cell subpopulation is unlikely. Fig. 4 demonstrates in the sample histograms from diverse autoimmune diseases that the major shift in MAD often
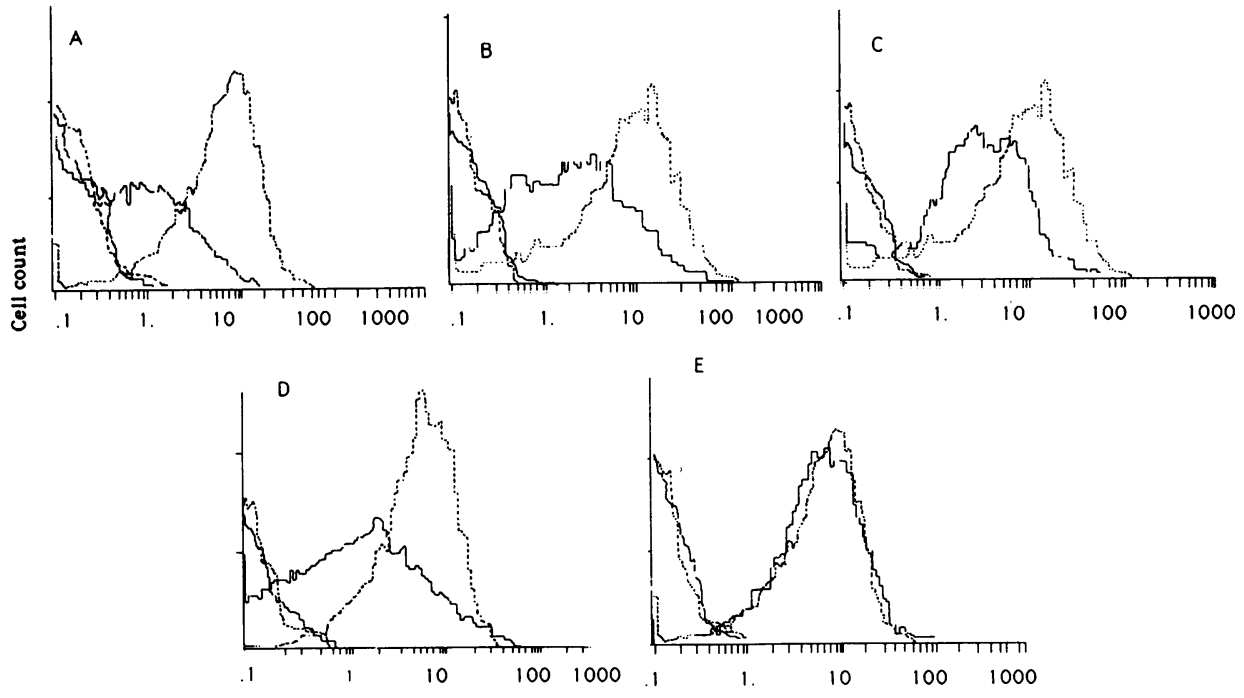

Fluorescence Intensity
Figure 4. MHC class I expression on EBV B cell lines from autoimmune patients (solid line) compared with control individuals (dashed line). $A$ IDDM; $B$, rheumatoid arthritis; $C$, lupus erythematosis; $D$, Graves'; $E$, noninsulin dependent diabetes (NIDDM). Background immunofluorescence is shown and was similar for all the cell lines. Each histogram pair represents a single characteristic patient and matched control. 
secondarily causes histogram overlap with background fluorescence and a resulting decrease in the percentage of positive cells. Therefore, the mean fluorescence as well as percentage of positive EBV cells for MHC class I are decreased in autoimmune diseases. As expected EBV cell lines from nonautoimmune NIDDM patients had a virtually identical MHC class I flow histogram to nondiabetic controls (Fig. $4 E$ ).

\section{Discussion}

Analysis of fresh lymphocytes and newly produced EBV-transformed B cell lines from Graves' disease, lupus erythematosis, Hashimoto's thyroiditis, Sjögren's syndrome, and rheumatoid arthritis patients revealed a uniform decrease in MHC class I expression on lymphoid cells not observed in control individuals or NIDDM, a chronic nonautoimmune disease. Similar to IDDM, a common finding in a variety of autoimmune diseases with strong genetic association with MHC class II linkage is a paradoxical defect in MHC class I expression that was present on all lymphocyte subpopulations and was most dramatically observed with a conformationally dependent class I antibody. We identified two NIDDM patients with decreased MHC class I expression on fresh PBL and EBV cell lines; follow-up autoantibody screening confirmed subclinical acute autoimmunity.

In light of the recent identification of new genes in the MHC class II region that influence MHC class I expression by supplying endogenous cytoplasmic peptides to MHC class I in the endoplasmic reticulum (8-15), it is interesting to speculate that, similar to IDDM, a common theme in autoimmunity is defective MHC class I function (8). Cell lines with induced mutations in these MHC class II-linked genes have defective MHC class I expression due to the faulty delivery of endogenous peptides associated with the abnormal MHC class II genes. This results in decreased class I assembly and surface expression. In the past, it has been difficult to explain how defective MHC class II could result in autoreactivity to self, since the known function of MHC class II is to present phagocytozed exogenous antigens to CD4 cells to elicit a positive immune response. The demonstration of a uniform decrease in MHC class I expression of lymphoid cells in autoimmune disease suggests that the presentation of endogenous peptides on MHC class I is a possible pathway for self-tolerance. Similar to transporter mutant cell lines, the defect in surface class I expression was most dramatically observed with a conformationally dependent antibody, thus implying that total surface class I is not only decreased in autoimmune lymphocytes but presumably peptide empty class $I$ is also present on the cell surface $(21$, 22 ). Interruption of this self-antigen presentation pathway, by any mechanism, is sufficient for autoreactivity manifested against a variety of target organs (8). A transgenic murine model with decreased MHC class I expression also develops autoimmunity (8).

A central unanswered question is how the target tissue of the various autoimmune diseases is specified. Perhaps the target of any given autoimmune disease is determined by nonMHG-linked genes determining target specificity. Alternatively, interruption of specific peptide supply factor genes in the MHC class II region in combination with a specific MHC class I haplotype may determine the target tissue most vulnerable to autoimmune attack. Further studies are required to establish the specific mechanisms by which MHC class I surface expression and presentation are altered in autoimmune disease.

\section{Acknowledgments}

We are indebted to Kathy Hurxthal, R.N., for her assistance in patient recruitment for blood donations and Lynne Murphy for her assistance in the preparation of the manuscript.

This work was supported by the Massachusetts General Hospital.

\section{References}

1. Cho, S., M. Attaya, and J. J. Monaco. 1991. New class II-like genes in the murine MHC. Nature (Lond.). 353:573-576.

2. Todd, J. A., J. I. Bell, and H. O. McDevitt. 1987. HLA-DQ ${ }_{\beta}$ gene contributes to susceptibility and resistance to insulin-dependent diabetes mellitus. $\mathrm{Na}$ ture (Lond.). 329:599-604.

3. Thomson, G., W. P. Robinson, M. K. Kuhner, S. Joe, M. J. MacDonald, J. L. Gottschall, J. Barbosa, S. S. Rich, J. Bertrams, and M. P. Baur. 1988. Genetic heterogeneity, modes of inheritance, and risk estimates for a joint study of Caucasians with insulin-dependent diabetes mellitus. Am. J. Hum. Genet. 43:799-816.

4. Baisch, J. M., T. Weeks, R. Giles, M. Hoover, P. Stastny, and J. D. Capra 1990. Analysis of HLA-DQ genotypes and susceptibility in insulin-dependent diabetes mellitus. $N$. Engl. J. Med. 322:1836-1841.

5. Moens, H., N. R. Farid, L. Sampson, E. P. Noel, and J. M. Barnard. 1978. Hashimoto's thyroiditis is associated with HLA-DRW3. N. Engl. J. Med. 299:133-134.

6. Svejgaard, A., P. Platz, and L. P. Ryder. 1983. HLA and disease 1982-A survey. Immunol. Rev. 70:193-218.

7. Maclaren, N. K., and W. J. Riley. 1986. Inherited susceptibility to autoimmune Addison's disease is linked to human leukocyte antigens-DR3 and/or DR4, except when associated with type I autoimmune polyglandular syndrome. J. Clin. Endocrin. \& Metab. 62:455-459.

8. Faustman, D., X. Li, H. Y. Lin, Y. Fu, G. Eisenbarth, J. Avruch, and J. Guo. 1991. Linkage of faulty major histocompatibility complex class I to autoimmune diabetes. Science (Wash. DC). 254:1756-1761.

9. Livingstone, A. M., S. J. Powis, A. G. Diamond, G. W. Butcher, and J. C. Howard. 1989. A trans-acting major histocompatibility complex-linked gene whose alleles determine gain and loss changes in the antigenic structure of a classical class I molecule. J. Exp. Med. 170:777-795.

10. Rotzschke, O., K. Falk, K. Deres, H. Schild, M. Narda, J. Metzger, G. Jung, and H. G. Rammensee. 1990. Isolation and analysis of naturally processed viral peptides as recognized by cytotoxic T-cells. Nature (Lond.). 348:252-254.

11. Spies, T., and R. DeMars. 1991. Restored expression of major histocompatibility class I molecules by gene transfer of a putative peptide transporter. Nature (Lond.). 351:323-324.

12. Townsend, A., C. Ohlen, J. Bastin, H. G. Ljunggren, L. Foster, and K. Karre. 1989. Association of class I major histocompatibility heavy and light chains induced by viral peptides. Nature (Lond.). 340:443-448.

13. Bevan, M. J. 1987. Class discrimination in the world of immunology. Nature (Lond.). 325:192-193.

14. Monaco, J. J., S. Cho, and M. Attaya. 1990. Transport protein genes in the murine MHC: Possible implications for antigen processing. Science (Wash. DC). 250:1723-1726.

15. Spies, T., M. Bresnahan, S. Bahrarn, D. Arnold, G. Blanck, E. Mellins, D. Plous, and R. DeMars. 1990. A gene in the human major histocompatibility complex class II region controlling the class I antigen presentation pathway. $\mathrm{Na}$ ture (Lond.). 348:744-747.

16. Trowsdale, J., I. Hanson, I. Mockridge, S. Beck, A. Townsend, and A. Kelly. 1990. Sequences encoded in the class II region of the MHC related to the 'ABC' superfamily of transporters. Nature (Lond.). 348:741-744.

17. Serra, H. M., J. A. Ledbetter, D. Neil, and L. M. Pilarski. 1988. Apparent Loss of $2 \mathrm{H} 4+\mathrm{T}$ cells in peripheral blood lymphocytes of normal donors: A 2H4-specific artifact unique to T cells. Hum. Immunol. 23:281-288.

18. Pirrucccello, S. J., M. Collins, J. E. Wilson, and B. M. McManus. 1989. Age-related changes in naive and memory $\mathrm{CD} 4+\mathrm{T}$ cells in healthy human children. Clin. Immunol. Immunopathol. 52:341-345.

19. Gaskins, H. R., J. J. Monaco, E. H. Leiter, L. S. Wicker, P. L. Podolin, P Fischer, A. Sirotina, R. C. Boltz, and L. B. Peterson. 1992. Expression of intraMHC transporter (HAM) genes and class I antigens in diabetes-susceptible NOD mice. Science (Wash. DC). 256:1826-1828.

20. Hokland, M., B. Larsen, I. Heron, and T. Plesner. 1981. Corticosteroids decrease the expression of $\beta_{2}$-microglobulin and histocompatibility antigens on human peripheral blood lymphocytes in vitro. Clin. Exp. Immunol. 44:239-246.

21. Catipovic, B., J. Dal Porto, M. Mage, T. E. Johansen, and J. P. Schneck. 1992. Major histocompatibility complex conformational epitopes are peptide specific. J. Exp. Med. 176:1611-1618.

22. Bluestone, J. A., S. Jameson, S. Miller, and R. Dick. 1992. Peptide-induced conformational changes in class I heavy chains alter major histocompatibility complex recognition. J. Exp. Med. 176:1757-1761. 